Neurosurg Focus 17 (4):E3, 2004

\title{
Efficacy of mechanical prophylaxis for venous thromboembolism in patients with brain tumors
}

\author{
Kurtis I. Auguste, M.D., Alfredo Quiñones-Hinojosa, M.D., \\ AND MitChel S. BERGER, M.D. \\ Department of Neurological Surgery, Brain Tumor Research Center, University of California, \\ San Francisco School of Medicine, San Francisco, California
}

\begin{abstract}
Patients with brain tumors are at considerable risk for the formation of venous thromboemboli. One method of preventing these complications is mechanical prophylaxis in which an external pneumatic compression device and graduated elastic compression stockings are used. Evidence indicates that these devices prevent deep venous thrombosis (DVT) and pulmonary embolism (PE) by limiting venous stasis and increasing fibrinolytic activity at both the local and systemic levels. The authors present evidence for the occurrence of both mechanisms and discuss the use of mechanical compression in the setting of surgery for brain tumors. They also present data proving the efficacy of these devices in patients who undergo craniotomy with motor mapping for resection of glioma and in whom the contralateral leg receives no prophylaxis. Finally, they comment on the use of anticoagulation therapy both in addition to and in place of mechanical prophylaxis.
\end{abstract}

\section{KEY WORDS • venous thromboembolism • external pneumatic compression device • compression stocking • craniotomy - motor mapping • glioma}

The incidence of DVT in neurosurgical patients ranges between 2 and 50\%.1,5,10,12,19,23,27,40,47,48,51,53 The rate of DVT and PE is thus quite significant and can lead to fatal outcomes. Neurosurgical procedures entail a 1.5 to $5 \%$ risk of $\mathrm{PE}$ and a 9 to $50 \%$ rate of mortality from this disorder. ${ }^{23}$ The prevention of VTE in neurosurgery by using mechanical prophylaxis is well established. Current modalities for mechanical prophylaxis involve pneumatic compression devices, graduated elastic compression stockings, or a combination of both. Prevention of VTE by using mechanical devices addresses tenets of the Virchow $^{54}$ triad: endothelial damage, venous stasis, and hypercoagulability. Mechanical prophylaxis appears to prevent thromboembolic complications by limiting venous stasis and diminishing hypercoagulability.

We provide a brief overview of the pathogenesis of thrombosis in patients undergoing neurosurgery and discuss the rationale for mechanical prophylaxis in the prevention of VTE. We also address a variation on mechanical prophylaxis in patients undergoing craniotomy with motor mapping. Finally, we comment briefly on the supplementation of mechanical prophylaxis with anticoagulation therapy.

\section{Hypercoagulability in the Postoperative Patient}

Neurosurgical patients are at considerable risk of ex-

Abbreviations used in this paper: $\mathrm{DVT}=$ deep venous thrombosis; $\mathrm{PE}=$ pulmonary embolism; $\mathrm{PA}=$ tissue plasminogen activator; $\mathrm{VTE}=$ venous thromboembolism. periencing thromboembolic complications. Neurological deficits caused either by the disease process or the procedures designed to treat it may limit patient activity and promote venous stasis in immobile extremities. Craniotomies performed for resection of malignant tumors have been proven to present an increased risk of VTE, $,, 51,53$ especially when the diagnosis is glioma. ${ }^{7,15,35,44}$ Malignancy alone has proven to be a significant risk factor for DVT and PE. ${ }^{18,28}$ Procedures for brain tumor resection are often time-consuming, and lengthy operations lead to prolonged venous stasis. ${ }^{48}$ The thromboembolic risks associated with neurosurgery cannot be ignored either in the operating room or during recovery.

The postoperative period is one marked by significant alterations in clotting enzymes. Patients undergoing surgical procedures have been noted to have increased inhibition of tPA, a potent clot-dissolving enzyme that converts plasminogen to plasmin (Fig. 1). ${ }^{28,32}$ Diminished tPA function will result in a reduced capacity for clearance of thrombi. Owen, et al. ${ }^{39}$ documented a marked imbalance between the proteolytic enzymes thrombin and plasmin in patients who underwent craniotomy. When this imbalance was allowed to persist, thrombi developed with increased frequency in these patients.

Localized hypoxemia within venous channels may further promote clot formation in the perioperative period. Hamer, et al., ${ }^{22}$ demonstrated that the valve pockets of veins become readily hypoxic during nonpulsatile flow. They demonstrated that diminished flow promoted early thrombus formation on the hypoxic valve cusps in as little as 2 hours. Lengthy neurosurgical procedures and the pro- 


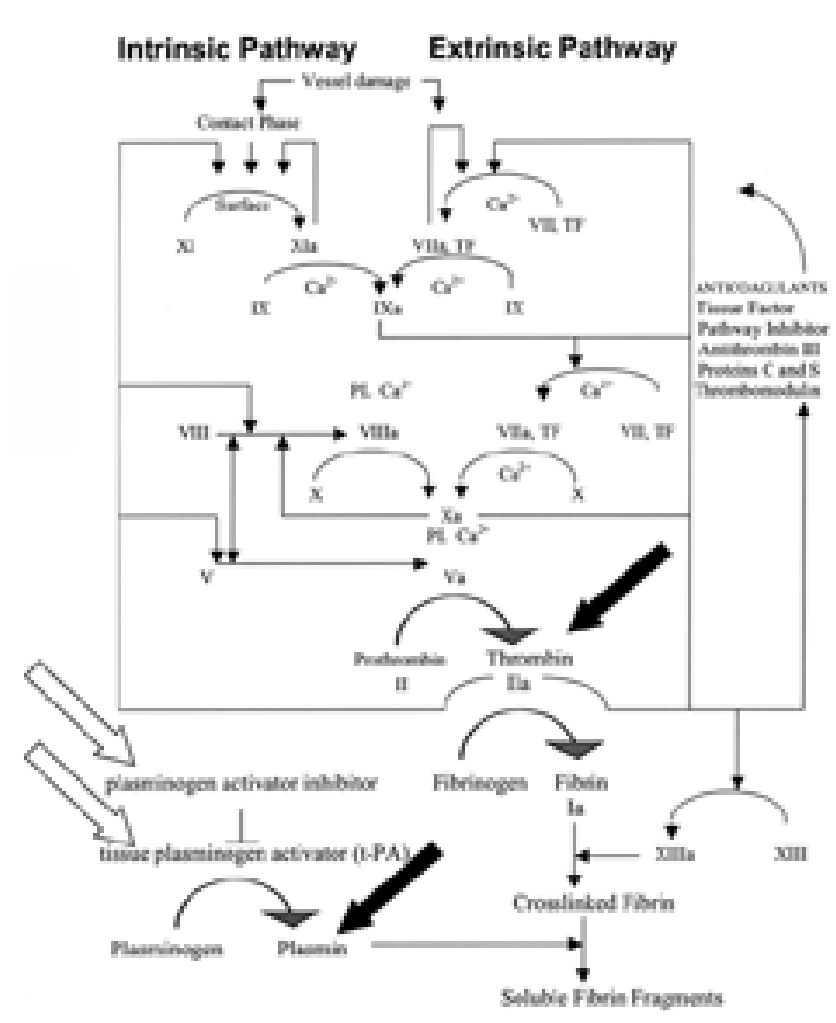

Fig. 1. Schematic diagram of the clotting cascade showing endothelial injury through both the intrinsic and extrinsic pathways. Mechanical prophylaxis has been shown to normalize the thrombin/plasmin ratio (black arrows), increase tPA levels, and decrease levels of plasminogen activator inhibitor (PAI; white arrows). Alterations in the levels of these proteolytic enzymes have been implicated in the reduced incidence of thromboembolism formation. Roman numerals denote clotting factors. PI = pathway inhibitor; $\mathrm{TF}=$ tissue factor.

longed inactivity associated with them are likely to reproduce these hypoxic conditions and predispose patients to clot formation.

\section{Pneumatic Compression Devices}

External pneumatic compression devices have been shown to prevent the formation of DVTs in neurosurgical patients. ${ }^{47,50,51}$ Modern devices evacuate blood from lower-extremity vessels in an automated fashion. In brief, a microprocessor directs pressurized air (for example, at 45 $\mathrm{mm} \mathrm{Hg}$ ) into segmental diaphragms secured around the leg for a fixed period of time (for example, for $11 \mathrm{sec}-$ onds). ${ }^{30}$ The compression is delivered in a sequential manner up the leg, producing a wavelike milking effect to evacuate leg veins. Sequential devices have been proven to be more effective than single-chamber, evenly distributed pressure in preventing DVTs. ${ }^{37}$ The compression is set to cycle regularly (for example, every 60 seconds). Devices are available for feet, calves, and/or thighs. Published contraindications for the use of these devices are listed in Table 1.

Pneumatic compression may exert its protective effect against thrombus formation in part by limiting venous stasis. Calnan, et al., ${ }^{11}$ were one of the first groups to illustrate that intermittent, rhythmic compression of the lower ex-
TABLE 1

Contraindications for the use of sequential pneumatic compression devices*

dermatitis

postop vein ligation

gangrene

recent skin graft

severe ischemic vascular disease

severe lower-extremity edema

extreme leg deformity

suspected preexisting DVT

* According to Jeffery and Nicolaides.

tremity mimics the normal pumping of calf muscles. Soon after, Sabri, et al., ${ }^{42}$ demonstrated a $400 \%$ increase in femoral vein pulsatility and a $250 \%$ increase in peak femoral venous blood flow when the devices were applied to the lower limbs of greyhound dogs. Comparable results were found in the lower extremities of humans who received pneumatic compression. ${ }^{41}$ In more modern studies investigators have shown increased blood flow velocities in the popliteal and common femoral veins by applying intermittent pneumatic compression to the foot alone. ${ }^{31}$ Mittelman, et al. ${ }^{36}$ compared the use of calf and thigh compression with calf compression alone and found an enhanced effect of blood clearance with sequential compressions of the calf and thigh. Similarly, Delis, et al., ${ }^{16}$ found greater outflow during foot plus calf compression when comparing it with foot compression alone. Current prophylactic devices most frequently compress at least two regions of the lower extremity, although more limited devices have retained some popularity.

Mechanical compression devices appear to exert part of their prophylactic effect through enhanced fibrinolysis. ${ }^{14,25,49}$ Early studies in which euglobulin clotting times were analyzed as a marker for systemic fibrinolysis activity in postoperative patients demonstrated that calf compression augments clot breakdown. ${ }^{3,33,43}$ Weitz, et al., 55 showed that intermittent pneumatic calf compression, by preserving the normal thrombin/plasmin ratio in blood samples obtained in patients who receive this therapy when compared with those not receiving pneumatic compression, averts the hypercoagulable state noted by Owen, et al. ${ }^{39}$ Intermittent pneumatic compression has been shown to increase the amount of tPA release and to decrease levels of plasminogen activator inhibitor. ${ }^{25}$ This benefit may be short-lived, however; diminished fibrinolytic activity is seen from several minutes ${ }^{25}$ to 18 hours $^{33}$ after discontinuation of pneumatic compression.

Various permutations of both upper- and lower-extremity compression have been tested to increase blood clearance and ultimately prevent VTEs. Nearly 30 years ago, Knight and Dawson ${ }^{33}$ conducted a study in which they applied intermittent compression to the upper extremities of patients who had undergone surgery, and noted a reduced incidence of DVTs in the legs and increased serum fibrinolytic activity. Tarnay, et al., ${ }^{49}$ also showed increased fibrinolysis in patients receiving compression in the arms, although this difference was not statistically significant. Another interesting finding of this study was the increased fibrinolysis detected in serum samples obtained in the pa- 
tient's arm after administration of pneumatic compression to the legs. This effect appeared to be proportional to the volume of tissue compressed; increased fibrinolysis was detected in patients who wore long compression boots when compared with those given shorter boots. The results of these studies indicate that the fibrinolytic activity promoted by mechanical prophylaxis has both local and systemic effects in protecting against clot formation.

\section{Compression Stockings}

An alternative or additional means of preventing thromboembolic complications is the graduated elastic compression stocking. 2,24,26,45 These devices apply continuous circumferential pressure to the lower extremity in a graduated fashion $(\sim 18 \mathrm{~mm} \mathrm{Hg}$ at the ankle to $\sim 8 \mathrm{~mm} \mathrm{Hg}$ at the thigh $)^{46}$ as a means of increasing venous clearance and preventing venous stasis. The stockings are contoured to the lower extremity to prevent tenting and focal constriction. Similar care should be exercised in selecting patients to receive stockings as is taken in choosing patients for treatment with pneumatic devices. The contraindications listed in Table 1 can be applied for stocking use as well.

In most neurosurgical practices, compression stockings are combined with pneumatic devices as a standard of care, presumably to enhance the prophylactic benefit of each. To test the theory that the protective effect of these devices is additive, Keith, et al., ${ }^{29}$ used Doppler ultrasonography imaging to measure the peak venous velocities produced in superficial femoral veins by the individual and simultaneous use of graduated compression stockings and intermittent pneumatic compression boots. Although their study was not designed to consider the effect on thrombosis formation, blood flow velocity served as a surrogate measure for the limited stasis and venous evacuation that could ultimately lead to the formation of thromboses. The results showed no synergistic effect on venous outflow when these devices were used simultaneously.

Two separate groups performed direct comparisons between compression stockings and pneumatic devices to assess the effectiveness of these modalities in reducing the risk of venous thrombosis. ${ }^{9,52}$ Based on their results, stockings appear to be as effective in VTE prevention as pneumatic compression devices, and stockings have the added benefit of lower cost. Patients also suffer less discomfort with stockings when compared with the pressure associated with intermittent leg compressions from pneumatic devices. In addition, the garments do not require assembly once fitted, are not subject to malfunction or troubleshooting, and facilitate patient mobility and ambulation during the postoperative recovery period.

Despite these findings, the combined approach in which both stockings and pneumatic devices are used remains popular, likely because of their relative ease of application or simply out of habit. Cost-conscious healthcare may limit their combined use in the future. In the meantime, practitioners should not be overly concerned when discomfort leads to discontinuation of pneumatic compression; these patients are not at any proven risk as long as they continue to use their compression stockings.

\section{Prophylaxis After Craniotomy for Motor Mapping}

Patients with lesions involving crucial speech or motor cortex present an added challenge in the prevention of VTE. Preservation of vital cortical structures necessitates intraoperative motor mapping and exposure of the contralateral lower extremity to monitor response to stimulation. The question then becomes: does craniotomy for motor mapping predispose neurosurgical patients to VTE formation?

To test this theory, we conducted a retrospective review of 188 patients who underwent craniotomy and motor mapping for glioma. ${ }^{4}$ In prior studies in which the use of mechanical prophylaxis for VTE was investigated, compression devices and stockings were routinely placed on both lower extremities. Given the scarcity of published data for unilateral prophylaxis, all patients included in this study received external pneumatic compression devices and compression stockings on the lower extremity ipsilateral to the side of the brain tumor and motor mapping. Patients with prior or current VTEs were excluded.

Our analysis indicated that motor mapping without contralateral mechanical prophylaxis does not place patients at a higher risk for VTE. We reported a $2.2 \%$ incidence of DVT and a $1.1 \%$ incidence of PE, which were comparable to statistics reported for neurosurgical patients receiving standard mechanical prophylaxis. A comprehensive review of this series showed no statistical significance for patient age, duration of surgery, or preoperative values for prothrombin and partial thromboplastin times (Table 2).

\section{Supplementary Anticoagulation Therapy}

The use of anticoagulation therapy during the perioperative period has been limited by concerns about intracranial bleeding. ${ }^{13,17}$ In a recent survey conducted among neurosurgeons, as many as $29 \%$ of respondents associated low-molecular-weight heparin with bleeding complications..$^{20}$ Added to this is the proven efficacy and safety of mechanical prophylaxis when compared directly with low-dose subcutaneously administered heparin. ${ }^{37,38} \mathrm{Nev}-$ ertheless, the use of perioperative anticoagulation therapy continues to have support and its safety in neurosurgical patients has been proven. ${ }^{1,6,12,21,34} \mathrm{~A}$ summary of these regimens is provided in Table 3.

The most reasonable solution for treatment concerns would be to tailor each prophylactic regimen to the individual. Patients with a higher than average thromboembolic risk warrant more aggressive prophylaxis. If the contraindications listed in Table 1 preclude the use of mechanical prophylaxis, anticoagulation therapy may be an appropriate supplementary or alternative treatment.

\section{CONCLUSIONS}

Mechanical prophylaxis for thromboemboli is an integral part of modern neurosurgical practice. It remains an effective means of preventing DVT and PE, both intraand postoperatively. Compression stockings and pneumatic devices are relatively equivalent in their prevention of VTE. Their effectiveness is not diminished when one leg is not treated with compression during motor mapping. Concomitant anticoagulation therapy during the postoperative period appears not only to be safe but also to protect patients further against thromboembolic complications. 
K. I. Auguste, A. Quiñones-Hinojosa, and M. S. Berger

TABLE 2

Demographic data in patients at UCSF who underwent craniotomy with motor mapping for glioma*

\begin{tabular}{|c|c|c|}
\hline Total No & o. w/ DVT/PE & No. w/o DVT \\
\hline no. of patients & 21 & 173 \\
\hline \multicolumn{3}{|l|}{ sex } \\
\hline male & 11 & 102 \\
\hline female & 10 & 71 \\
\hline \multicolumn{3}{|l|}{ resection } \\
\hline primary & 12 & 96 \\
\hline revision & 9 & 77 \\
\hline \multicolumn{3}{|l|}{ mapping } \\
\hline motor & 21 & 166 \\
\hline motor \& speech & 0 & 7 \\
\hline \multicolumn{3}{|l|}{ side of op } \\
\hline $\mathrm{rt}$ & 12 & 118 \\
\hline lt & 9 & 55 \\
\hline \multicolumn{3}{|l|}{ habits } \\
\hline smoking & 4 & 44 \\
\hline alcohol & 0 & 26 \\
\hline IV drug use & 0 & 1 \\
\hline \multicolumn{3}{|l|}{$\mathrm{CV}$ risk factors } \\
\hline CV disease & 6 & 32 \\
\hline HTN & 4 & 25 \\
\hline valve disease & 2 & 0 \\
\hline high lipid levels & 0 & 3 \\
\hline high cholesterol & 0 & 11 \\
\hline previous op & 16 & 149 \\
\hline intracranial & 11 & 102 \\
\hline extracranial & 5 & 47 \\
\hline anticoagulant drugs on admit & 0 & $\begin{array}{l}\text { coumadin, 2; LMW heparin, } \\
2 \text {; aspirin, } 3 \text {; other NSAIDs, } 2\end{array}$ \\
\hline \multicolumn{3}{|l|}{ intraop DVT prophylaxis } \\
\hline SCD & 19 & 0 \\
\hline PCD & 0 & 153 \\
\hline stockings & 21 & 160 \\
\hline side specified & 7 & 93 \\
\hline side not specified & 14 & 80 \\
\hline \multicolumn{3}{|l|}{$\mathrm{dx}$} \\
\hline DVT & 20 & NA \\
\hline $\mathrm{PE}$ & 7 & NA \\
\hline \multicolumn{3}{|l|}{ method of DVT dx } \\
\hline US & 5 & NA \\
\hline$H \& P$ & 1 & NA \\
\hline unknown & 15 & NA \\
\hline \multicolumn{3}{|l|}{ method of PE dx } \\
\hline helical CT & 2 & NA \\
\hline presumed hypoxemia + DVT & 1 & NA \\
\hline unknown & 14 & NA \\
\hline \multicolumn{3}{|l|}{ side of DVT } \\
\hline w/o prophylaxis only & 7 & NA \\
\hline w/ prophylaxis only & 2 & NA \\
\hline both sides w/ prophylaxis & 1 & NA \\
\hline bilat DVTs & 4 & NA \\
\hline $\begin{array}{l}\text { prophylaxis unspecified but } \\
\text { DVT contralat to op side }\end{array}$ & 3 & NA \\
\hline DVT side unstated & 2 & NA \\
\hline \multicolumn{3}{|l|}{ follow-up time $(\mathrm{mos}) \dagger$} \\
\hline mean & 11.7 & 13.5 \\
\hline median & 9.0 & 9.0 \\
\hline other risk factors & & $\begin{array}{l}\text { nephrotic syndrome }+ \\
\text { vasculitis, } 1 \text {; OCPs, } 2 \text {; } \\
\text { recent trauma, } 4\end{array}$ \\
\hline
\end{tabular}

* Portions of these data were previously analyzed and included in a prior publication (Auguste, et al.). Abbreviations: $\mathrm{CT}=$ computerized tomography; $\mathrm{CV}=$ cardiovascular; $\mathrm{dx}=$ diagnosis; $\mathrm{H} \& \mathrm{P}=$ history and physical examination; HTN = hypertension; IV = intravenous; LMW = low-molecular-weight; NA = not applicable; NSAID = nonsteroidal antiinflammatory drug; $\mathrm{OCP}=$ oral contraceptive pill; $\mathrm{PCD}=$ pneumatic compression device; $\mathrm{SCD}=$ sequential compression device; UCSF = University of California, San Francisco; US = ultrasound.

† Follow up for all patients: less than 3 months, 24\%; 3 to 6 months, $12.4 \%$; 6 to 9 months, $13.8 \%$; 9 to 12 months, $9.5 \%$; 1 to 2 years, $20.4 \%$; 2 to 3 years, $13.9 \%$; 3 to 4 years, $4.4 \%$; more than 4 years, $1.4 \%$.
TABLE 3

Literature review of evidence-based regimens for safe anticoagulation therapy in patients undergoing neurosurgery*

\begin{tabular}{lll}
\hline \hline \multicolumn{1}{c}{ Authors \& Year } & Anticoagulating Agent & \multicolumn{1}{c}{ Regimen } \\
\hline Cerrato, et al., 1978 & heparin & $5000 \mathrm{U}$ Q 8 hrs \\
Bostrom, et al., 1986 & heparin & $5000 \mathrm{U}$ Q 12 hrs \\
Agnelli, et al., 1998 & enoxaparin & $40 \mathrm{mg}$ QD \\
Macdonald, et al., 1999 & heparin & $5000 \mathrm{U}$ Q 12 hrs \& \\
& & $5000 \mathrm{U}$ Q 8 hrs \\
Goldhaber, et al., 2002 & enoxaparin & $40 \mathrm{mg}$ QD \\
\hline * QD = every day. & &
\end{tabular}

\section{References}

1. Agnelli G, Piovella F, Buoncristiani P, et al: Enoxaparin plus compression stockings compared with compression stockings alone in the prevention of venous thromboembolism after elective neurosurgery. N Engl J Med 339:80-85, 1998

2. Allan A, Williams JT, Bolton JP, et al: The use of graduated compression stockings in the prevention of postoperative deep vein thrombosis. Br J Surg 70:172-174, 1983

3. Allenby F, Boardman L, Pflug JJ, et al: Effects of external pneumatic intermittent compression on fibrinolysis in man. Lancet 2:1412-1414, 1973

4. Auguste KI, Quinones-Hinojosa A, Gadkary C, et al: Incidence of venous thromboembolism in patients undergoing craniotomy and motor mapping for glioma without intraoperative mechanical prophylaxis to the contralateral leg. J Neurosurg 99: 680-684, 2003

5. Becker DM, Gonzalez M, Gentili A, et al: Prevention of deep venous thrombosis in patients with acute spinal cord injuries: use of rotating treatment tables. Neurosurgery 20:675-677, 1987

6. Bostrom S, Holmgren E, Jonsson O, et al: Post-operative thromboembolism in neurosurgery. A study on the prophylactic effect of calf muscle stimulation plus dextran compared to low-dose heparin. Acta Neurochir 80:83-89, 1986

7. Brandes AA, Scelzi E, Salmistraro G, et al: Incidence of risk of thromboembolism during treatment high-grade gliomas: a prospective study. Eur J Cancer 33:1592-1596, 1997

8. Brisman R, Mendell J: Thromboembolism and brain tumors. J Neurosurg 38:337-338, 1973

9. Bucci MN, Papadopoulos SM, Chen JC, et al: Mechanical prophylaxis of venous thrombosis in patients undergoing craniotomy: a randomized trial. Surg Neurol 32:285-288, 1989

10. Bynke O, Hillman J, Lassvik C: Does peroperative external pneumatic leg muscle compression prevent post-operative venous thrombosis in neurosurgery? Acta Neurochir 88:46-48, 1987

11. Calnan JS, Pflug JJ, Mills CJ: Pneumatic intermittent-compression legging simulating calf-muscle pump. Lancet 2:502-503, 1970

12. Cerrato D, Ariano C, Fiacchino F: Deep vein thrombosis and low-dose heparin prophylaxis in neurosurgical patients. J Neurosurg 49:378-381, 1978

13. Clagett GP, Anderson FA Jr, Heit J, et al: Prevention of venous thromboembolism. Chest 108 (Suppl 4):312S-334S, 1995

14. Comerota AJ, Chouhan V, Harada RN, et al: The fibrinolytic effects of intermittent pneumatic compression: mechanism of enhanced fibrinolysis. Ann Surg 226:306-314, 1997

15. Constantini S, Kornowski R, Pomeranz S, et al: Thromboembolic phenomena in neurosurgical patients operated upon for primary and metastatic brain tumours. Acta Neurochir 109: 93-97, 1991

16. Delis KT, Slimani G, Hafez HM, et al: Enhancing venous outflow in the lower limb with intermittent pneumatic compres- 
sion. A comparative haemodynamic analysis on the effect of foot vs. calf vs. foot and calf compression. Eur J Vasc Endovasc Surg 19:250-260, 2000

17. Dickinson LD, Miller LD, Patel CP, et al: Enoxaparin increases the incidence of postoperative intracranial hemorrhage when initiated preoperatively for deep venous thrombosis prophylaxis in patients with brain tumors. Neurosurgery 43:1074-1081, 1998

18. Francis JL, Biggerstaff J, Amirkhosravi A: Hemostasis and malignancy. Semin Thromb Hemost 24:93-109, 1998

19. Frim DM, Barker FG II, Poletti CE, et al: Postoperative lowdose heparin decreases thromboembolic complications in neurosurgical patients. Neurosurgery 30:830-833, 1992

20. Gnanalingham KK, Holland JP: Attitudes to the use of prophylaxis for thrombo-embolism in neurosurgical patients. J Clin Neurosci 10:467-469, 2003

21. Goldhaber SZ, Dunn K, Gerhard-Herman M, et al: Low rate of venous thromboembolism after craniotomy for brain tumor using multimodality prophylaxis. Chest 122:1933-1937, 2002

22. Hamer JD, Malone PC, Silver IA: The PO2 in venous valve pockets: its possible bearing on thrombogenesis. Br J Surg 68: 166-170, 1981

23. Hamilton MG, Hull RD, Pineo GF: Venous thromboembolism in neurosurgery and neurology patients: a review. Neurosurgery 34:280-296, 1994

24. Holford CP: Graded compression for preventing deep venous thrombosis. Br Med J 2:969-970, 1976

25. Jacobs DG, Piotrowski JJ, Hoppensteadt DA, et al: Hemodynamic and fibrinolytic consequences of intermittent pneumatic compression: preliminary results. J Trauma 40:710-717, 1996

26. Jeffery PC, Nicolaides AN: Graduated compression stockings in the prevention of postoperative deep vein thrombosis. Br J Surg 77:380-383, 1990

27. Joffe SN: Incidence of postoperative deep vein thrombosis in neurosurgical patients. J Neurosurg 42:201-203, 1975

28. Juhan-Vague I, Moerman B, De Cock F, et al: Plasma levels of a specific inhibitor of tissue-type plasminogen activator (and urokinase) in normal and pathological conditions. Thromb Res 33:523-530, 1984

29. Keith SL, McLaughlin DJ, Anderson FA Jr, et al: Do graduated compression stockings and pneumatic boots have an additive effect on the peak velocity of venous blood flow? Arch Surg 127:727-730, 1992

30. Kendall Healthcare Products Company: Operation \& Service Manual: Model 6325. SCD Sequel Compression System. Mansfield, MA: Tyco International Ltd., 1995

31. Killewich LA, Sandager GP, Nguyen AH, et al: Venous hemodynamics during impulse foot pumping. J Vasc Surg 22: 598-605, 1995

32. Kluft C, Verheijen JH, Jie AF, et al: The postoperative fibrinolytic shutdown: a rapidly reverting acute phase pattern for the fast-acting inhibitor of tissue-type plasminogen activator after trauma. Scand J Clin Lab Invest 45:605-610, 1985

33. Knight MT, Dawson R: Effect of intermittent compression of the arms on deep venous thrombosis in the legs. Lancet 2: $1265-1268,1976$

34. Macdonald RL, Amidei C, Baron J, et al: Randomized, pilot study of intermittent pneumatic compression devices plus dalteparin versus intermittent pneumatic compression devices plus heparin for prevention of venous thromboembolism in patients undergoing craniotomy. Surg Neurol 59:363-374, 2003

35. Marras LC, Geerts WH, Perry JR: The risk of venous thromboembolism is increased throughout the course of malignant glioma: an evidence-based review. Cancer 89:640-646, 2000

36. Mittelman JS, Edwards WS, McDonald JB: Effectiveness of leg compression in preventing venous stasis. Am J Surg 144: 611-613, 1982

37. Nicolaides AN, Fernandes e Fernandes J, Pollock AV: Intermittent sequential pneumatic compression of the legs in the pre- vention of venous stasis and postoperative deep venous thrombosis. Surgery 87:69-76, 1980

38. Nicolaides AN, Miles C, Hoare M, et al: Intermittent sequential pneumatic compression of the legs and thromboembolismdeterrent stockings in the prevention of postoperative deep venous thrombosis. Surgery 94:21-25, 1983

39. Owen J, Kvam D, Nossel HL, et al: Thrombin and plasmin activity and platelet activation in the development of venous thrombosis. Blood 61:476-482, 1983

40. Powers SK, Edwards MS: Prophylaxis of thromboembolism in the neurosurgical patient: a review. Neurosurgery 10: 509-513, 1982

41. Roberts VC, Sabri S, Beeley AH, et al: The effect of intermittently applied external pressure on the haemodynamics of the lower limb in man. Br J Surg 59:223-226, 1972

42. Sabri S, Roberts VC, Cotton LT: The effects of intermittently applied external pressure on the haemodynamics of the hindlimb in greyhound dogs. Br J Surg 59:219-222, 1972

43. Sabri S, Roberts VC, Cotton LT: Prevention of early postoperative deep vein thrombosis by intermittent compression of the leg during surgery. Br Med J 4:394-396, 1971

44. Sawaya R, Zuccarello M, Elkalliny M, et al: Postoperative venous thromboembolism and brain tumors: Part I. Clinical profile. J Neurooncol 14:119-125, 1992

45. Scurr JH, Ibrahim SZ, Faber RG, et al: The efficacy of graduated compression stockings in the prevention of deep vein thrombosis. Br J Surg 64:371-373, 1977

46. Sigel B, Edelstein AL, Savitch L, et al: Type of compression for reducing venous stasis. A study of lower extremities during inactive recumbency. Arch Surg 110:171-175, 1975

47. Skillman JJ, Collins RE, Coe NP, et al: Prevention of deep vein thrombosis in neurosurgical patients: a controlled, randomized trial of external pneumatic compression boots. Surgery 83: 354-358, 1978

48. Swann KW, Black PM: Deep vein thrombosis and pulmonary emboli in neurosurgical patients: a review. J Neurosurg 61: 1055-1062, 1984

49. Tarnay TJ, Rohr PR, Davidson AG, et al: Pneumatic calf compression, fibrinolysis, and the prevention of deep venous thrombosis. Surgery 88:489-496, 1980

50. Turpie AG, Delmore T, Hirsh J, et al: Prevention of venous thrombosis by intermittent sequential calf compression in patients with intracranial disease. Thromb Res 15:611-616, 1979

51. Turpie AG, Gallus A, Beattie WS, et al: Prevention of venous thrombosis in patients with intracranial disease by intermittent pneumatic compression of the calf. Neurology 27:435-438, 1977

52. Turpie AG, Hirsh J, Gent M, et al: Prevention of deep vein thrombosis in potential neurosurgical patients. A randomized trial comparing graduated compression stockings alone or graduated compression stockings plus intermittent pneumatic compression with control. Arch Intern Med 149:679-681, 1989

53. Valladares JB, Hankinson J: Incidence of lower extremity deep vein thrombosis in neurosurgical patients. Neurosurgery 6: 138-141, 1980

54. Virchow RLK: Thrombose and Emboli. Frankfurt: Barth, 1856

55. Weitz J, Michelsen J, Gold K, et al: Effects of intermittent pneumatic calf compression on postoperative thrombin and plasmin activity. Thromb Haemost 56:198-201, 1986

Manuscript received August 24, 2004.

Accepted in final form September 7, 2004.

Address reprint requests to: Kurtis Ian Auguste, M.D., Department of Neurological Surgery, University of California, San Francisco, 505 Parnassus Avenue, M-779, San Francisco, California 94143-0112. email: augustek@ neurosurg.ucsf.edu. 